\title{
BMJ Open Cost of maternal health services in low and middle-income countries: protocol for a systematic review
}

\author{
Aduragbemi Banke-Thomas, ${ }^{\oplus 1}$ Ibukun-Oluwa Omolade Abejirinde, ${ }^{\circ} 2$ \\ Oluwasola Banke-Thomas, ${ }^{3}$ Adamu Maikano, ${ }^{4}$ Charles Anawo Ameh ${ }^{\odot 5}$
}

To cite: Banke-Thomas $A$ Abejirinde I-00, BankeThomas 0 , et al. Cost of maternal health services in low and middle-income countries: protocol for a systematic review. BMJ Open 2019;9:e027822. doi:10.1136/ bmjopen-2018-027822

- Prepublication history and additional material for this paper are available online. To view these files, please visit the journal online (http://dx.doi. org/10.1136/bmjopen-2018027822).

Received 08 November 2018

Revised 29 June 2019 Accepted 01 August 2019

Check for updates

(C) Author(s) (or their employer(s)) 2019. Re-use permitted under CC BY-NC. No commercial re-use. See rights and permissions. Published by BMJ.

${ }^{1}$ Department of Health Policy, London School of Economics and Political Science, London, United Kingdom

${ }^{2}$ Center for Global Child Health, The Hospital for Sick Children (SickKids), Toronto, Ontario, Canada

${ }^{3}$ Southwest Interdisciplinary Research Center, Arizona State University, Phoenix, Arizona, USA ${ }^{4}$ Department of Prevention and Community Programmes, APIN Public Health Initiatives, Jabi District, Abuja, Nigeria

${ }^{5}$ Centre for Maternal and

Newborn Health, Liverpool

School of Tropical Medicine, Liverpool, United Kingdom

Correspondence to

Dr Aduragbemi Banke-Thomas; a.banke-thomas@Ise.ac.uk

\section{ABSTRACT}

Introduction There is substantial evidence that maternal health services across the continuum of care are effective in reducing morbidities and mortalities associated with pregnancy and childbirth. There is also consensus regarding the need to invest in the delivery of these services towards the global goal of achieving Universal Health Coverage in low/middle-income countries (LMICs). However, there is limited evidence on the costs of providing these services. This protocol describes the methods and analytical framework to be used in conducting a systematic review of costs of providing maternal health services in LMICs.

Methods African Journal Online, CINAHL Plus, EconLit, Embase, Global Health Archive, Popline, PubMed and Scopus as well as grey literature databases will be searched for relevant articles which report primary cost data for maternal health service in LMICs published from January 2000 to June 2019. This search will be conducted without implementing any language restrictions. Two reviewers will independently search, screen and select articles that meet the inclusion criteria, with disagreements resolved by discussions with a third reviewer. Quality assessment of included articles will be conducted based on cost-focused criteria included in globally recommended checklists for economic evaluations. For comparability, where feasible, cost will be converted to international dollar equivalents using purchasing power parity conversion factors. Costs associated with providing each maternal health services will be systematically compared, using a subgroup analysis. Sensitivity analysis will also be conducted. Where heterogeneity is observed, a narrative synthesis will be used. Population contextual and intervention design characteristics that help achieve cost savings and improve efficiency of maternal health service provision in LMICs will be identified.

Ethics and dissemination Ethical approval is not required for this review. The plan for dissemination is to publish review findings in a peer-reviewed journal and present findings at high-level conferences that engage the most pertinent stakeholders.

PROSPERO registration number CRD42018114124

\section{INTRODUCTION}

In September 1990, world leaders gathered at the United Nations General Assembly to launch the Millennium Development Goals

\section{Strengths and limitations of this study}

To the best of our knowledge, this protocol provides a detailed description of the first systematic review on cost of maternal health services conducted since year 2000

- The protocol adheres to the Preferred Reporting Items for Systematic Reviews and Meta-Analyses Protocols guidelines for reporting a systematic review protocol.

- The protocol is being conducted by a multidisciplinary team with experience conducting similar cost-based systematic reviews.

- Possibility that not all relevant costing studies of maternal health services will be retrieved, especially those in the grey literature.

(MDGs), one of which focused on improving maternal health (goal 5). This goal aimed to reduce maternal mortality ratio (MMR) by three quarters, between 1990 and 2015 . Despite concerted efforts which led to a $44 \%$ global reduction in MMR by the end of the MDG era, 303000 women still die every year due to complications associated with pregnancy and childbirth, ${ }^{1}$ with $99 \%$ of these occurring in low/middle-income countries (LMICs). In addition, 2.6 million babies die before they are born $50 \%$ of them in the third trimester) and 2.7 million die within the first month of life. ${ }^{2}$ The challenge to reduce these preventable deaths remains in the Sustainable Development Goals (SDGs) era in which the target is the reduce MMR to 70 deaths per 100000 live births globally. ${ }^{34}$

There is evidence to the effectiveness of critical care packages (antenatal care (ANC), skilled birth attendance, Emergency Obstetric Care (EmOC) (including injectable antibiotics, injectable oxytocics, injectable anticonvulsants, manual removal of placenta, removal of retained products, assisted vaginal delivery, basic neonatal resuscitation, caesarean section and blood transfusion), postnatal care (PNC) and family planning) 
Table 1 Definition of key terms relevant to the systematic review

\begin{tabular}{ll}
\hline Key terms & Definitions \\
\hline Antenatal care (ANC) & $\begin{array}{l}\text { Care provided by skilled healthcare professionals to pregnant women in order to ensure the best health } \\
\text { conditions for both mother and baby during pregnancy. }\end{array}$ \\
$\begin{array}{l}\text { Skilled birth } \\
\text { attendance }\end{array}$ & $\begin{array}{l}\text { Delivery by a health professional who can identify and manage normal labour and delivery; and identify } \\
\text { and treat complications or provide basic care and referral conducted within an enabling environment. }\end{array}$ \\
$\begin{array}{l}\text { Emergency obstetric } \\
\text { care (EmOC) }\end{array}$ & $\begin{array}{l}\text { Care package required to treat complications that arise from pregnancy and childbirth. There are } \\
\text { two levels of care (basic and comprehensive). Basic EmOC includes parenteral administration of } \\
\text { parenteral antibiotics, uterotonic drugs and parenteral anticonvulsants, manual removal of placenta, } \\
\text { removal of retained products, and performance of assisted vaginal delivery. At a higher level of care, } \\
\text { comprehensive EmOC includes all Basic EmOC interventions, blood transfusion and caesarean } \\
\text { section services. }\end{array}$ \\
Postnatal care & $\begin{array}{l}\text { Care given to the mother immediately after the birth and for the first six weeks of life. } \\
\text { Services including educational, comprehensive medical or social activities which enable individuals, } \\
\text { Family planning } \\
\text { services }\end{array}$ \\
including minors, to determine freely the number and spacing of their children and to select the means \\
by which this may be achieved (For this review, focus will be on women).
\end{tabular}

in reducing maternal morbidity and mortality. ${ }^{5-10}$ More so, when combined and integrated as a continuum of care. $^{11} 12$ Definitions of the maternal health services covered in this review are presented in table 1 .

In addition to the extensive literature on the effectiveness of these care packages, there is also wide consensus on the economic benefits of investing in maternal health services across the continuum of care, especially as healthier women and their children contribute to more productive and sustainable societies. ${ }^{12-14}$ Evidence also suggests that maternal health services especially those that are preventive in nature such as ANC are highly cost-effective. ${ }^{15}$ However, there is limited data on the cost of providing the services in LMICs. Tools such as the WHO-CHOICE (CHOosing Interventions that are Cost Effective) have attempted to collect costs estimates for health services more broadly. ${ }^{1617}$ This tool is based on predictions made from modelling of primary and secondary data and is not devoid of its flaws. Furthermore, WHO-CHOICE does not capture all costs related to maternal health services, such as the cost of medicines, and the costs covered are not specific for maternal health. ${ }^{18}$ Use of robust country-specific data collected from representative populations are therefore preferred for costing health services, ${ }^{19}$ more so maternal health services. ${ }^{20}$

To efficiently and effectively provide maternal health services, skilled health workers, functional equipment, adequate medicines and supplies are required, all of which have attributable costs, irrespective of the facility ownership (public, private or mission owned). Despite low gross domestic product per capita income in LMICs (defined by the World Bank as <US\$3385 in July 2018), ${ }^{21}$ governments traditionally provide the majority of funding required for maternal health services. This is complemented by private and third sector organisations (including non-governmental organisations, charities and missionaries). On the other hand, women using the services also incur costs associated with their use of maternal health services. Costs are often times a barrier for women living in LMICs to access necessary maternal health services. ${ }^{22}$
Data on costs of these services are therefore needed to complement the already established effectiveness data and facilitate the conduct of cost-effectiveness and value-for-money studies more broadly. ${ }^{23}$ Such data will also feed into priority setting and resource allocation for maternal health in LMICs. However, a preliminary search of PubMed, Scopus, Embase, Cochrane database, Joanna Briggs Institute Database of Systematic Reviews and Implementation Reports and PROSPERO to identify systematic reviews that had been conducted on this topic area revealed only one previous review. The identified previously conducted review was published in the year 2000 and included papers published mostly in the 1990 s. $^{20}$ In the SDG era, where competition for limited resources is high, evidence on the cost of providing maternal health services will be central to informing policy and practice. ${ }^{423}$ The objective of this review is to assess the costs associated with maternal health services in LMICs from available evidence in the peer-reviewed and grey literature. To achieve this objective, the following research questions were developed:

1. What are the costs associated with the provision of maternal health services in LMICs?

a. What cost items for various maternal health services have been reported in the literature?

b. How have such cost data been collected and analyzed in the existing body of literature?

c. What are the similarities and differences in the cost of providing maternal health services in LMICs?

2. What lessons can be learnt from different cost-saving techniques used in providing maternal health services in LMICs?

\section{METHODS}

\section{Protocol registration}

This protocol is registered in the PROSPERO database. In designing the proposed methods for the review, we leveraged best practices for conducting systematic reviews 


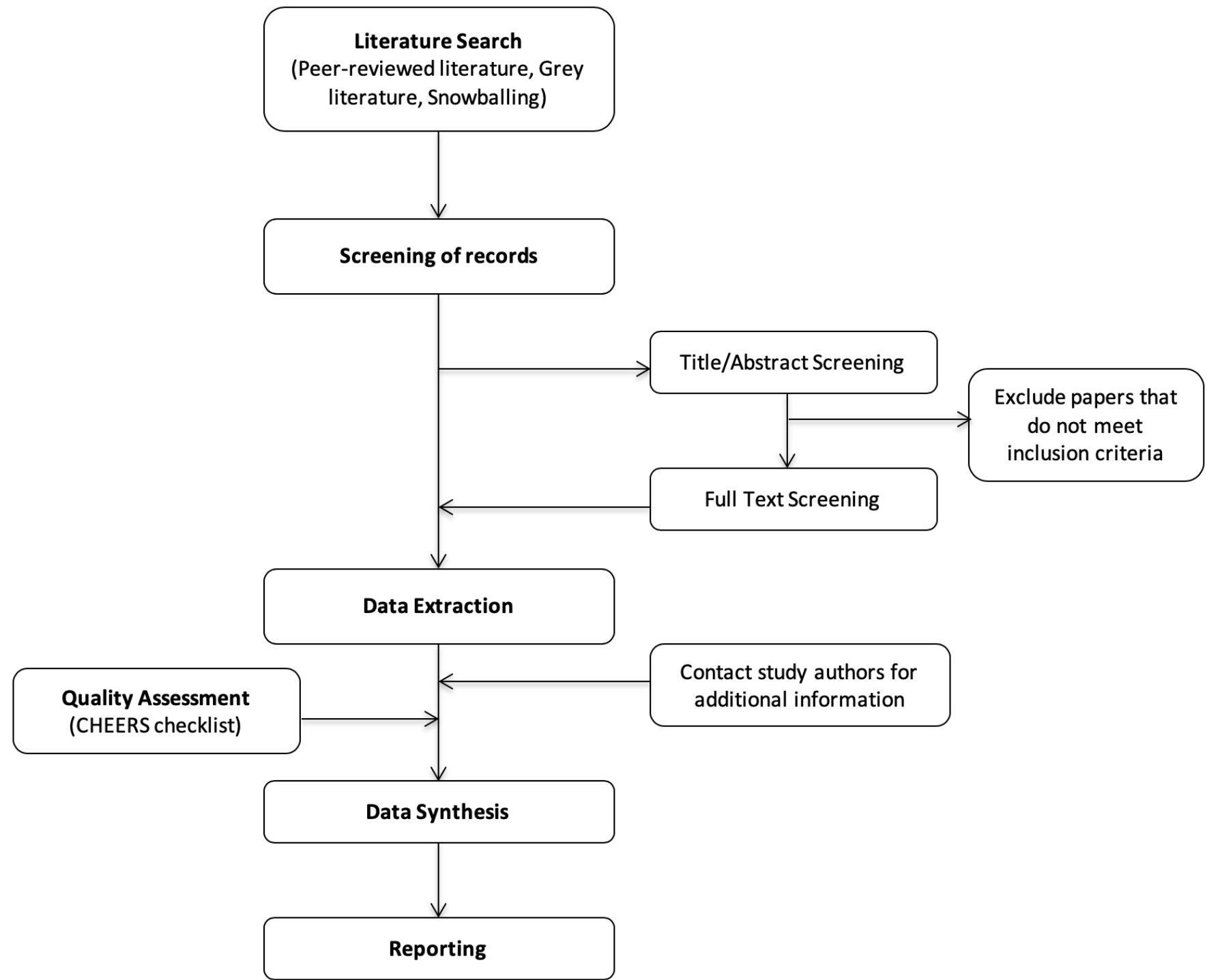

Figure 1 Summary of search strategy search process. CHEERS, Consolidated Health Economic Evaluation Reporting Standards.

on costs and cost-effectiveness of interventions from the Centre for Reviews and Dissemination and the Task Force on Community Preventive Services. ${ }^{24} 25$ The protocol adheres to the Preferred Reporting Items for Systematic Reviews and Meta-Analyses Protocols (PRISMA-P) 2015. ${ }^{26}$ PRISMA-P $2015^{26}$ (see online supplementary material S1).

\section{Patient and public involvement}

Patients and the public were not involved in the design of this systemic review protocol.

\section{Study design}

A systematic review of peer review and grey literature following the Preferred Reporting Items for Systematic Reviews and Meta-Analyses (PRISMA) approach by Moher et $a l^{27}$ is planned for this review. Figure 1 summarises the planned stages of the review as described in this protocol.

\section{Data source and search strategy}

A preliminary search was conducted on 2 January 2019 to test the predesigned search strategy. A repeat search will be conducted 30 June 2019 to bring our review up to date before publication and ensure that no recent relevant articles will be missed. We will search multiple databases: African Journal Online, CINAHL Plus, EconLit, Embase, Global Health Archive, Popline, PubMed and Scopus. In searching the various databases, and where relevant, we will combine medical subject headings and/or keywords, using Boolean linkages 'OR' within categories and 'AND' between three groups of words and phrases that capture the interventions, costs and the setting of interest-LMICs. Table 2 shows a summary of the search strategy that will be adapted for the various databases. The combination of these search terms guarantees an optimal search strategy for retrieving cost and economic studies relevant to maternal health services, ${ }^{28}$ and has been 
Table 2 Search terms to be adapted for use in the different databases for the review

\begin{tabular}{|c|c|}
\hline $\begin{array}{l}\text { Search term } \\
\text { category }\end{array}$ & Specific search term \\
\hline Intervention & $\begin{array}{l}\text { 'ante*natal care' OR ANC OR ‘birth' OR 'skilled birth attendance' OR 'obstetric emergenc*' OR 'emergency } \\
\text { obstetric care' OR EmOC OR 'caesarean*' OR ‘vacuum' OR 'post*natal care' OR 'PNC' OR obstetric OR } \\
\text { delivery OR maternity OR 'family planning' OR contraception }\end{array}$ \\
\hline Cost & 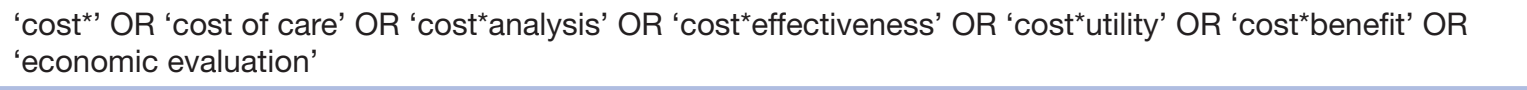 \\
\hline $\begin{array}{l}\text { Setting of } \\
\text { interest }\end{array}$ & $\begin{array}{l}\text { 'Low-and-Middle-Income Countr’, 'Iow income countr”, 'Africa', 'sub-Saharan Africa', 'Asia', Afghanistan, } \\
\text { Albania, Algeria, Angola, Argentina, Armenia, Azerbaijan Bangladesh, Belarus, Belize, Benin, Bhutan, Bolivia, } \\
\text { 'Bosnia and Herzegovina', Botswana, 'Brazil', 'Burkina Faso', Burundi, 'Cabo Verde', Cambodia, Cameroon, } \\
\text { 'Central African Republic', Chad, China, Colombia, Comoros, 'Democratic Republic of Congo', Congo, } \\
\text { 'Costa Rica', 'Côte d'Ivoire', Cuba, Djibouti, Dominica, 'Dominican Republic', Ecuador, Egypt, 'El Salvador', } \\
\text { 'Equatorial Guinea', Eritrea, Eswatini, Ethiopia, Fiji, Gabon, Gambia, Georgia, Ghana, Grenada, Guatemala, } \\
\text { Guinea, Guinea-Bissau, Guyana, Haiti, Honduras, India, Indonesia, Iran, Iraq, Jamaica, Kazakhstan, Kenya, } \\
\text { Kiribati, 'Democratic People’s Republic of Korea', Kosovo, Kyrgyzstan, 'Lao People's Democratic Republic', } \\
\text { Lebanon, Lesotho, Liberia, Libya, Madagascar, Malawi, Maldives, Mali, 'Marshall Islands', Mauritania, } \\
\text { Maritius, Mexico, Micronesia, Moldova, Mongolia, Montenegro, Morocco, Mozambique, Myanmar, Namibia, } \\
\text { Nauru, Nepal, Nicaragua, Niger, Nigeria, North Macedonia, Pakistan, Palau, Panama, 'Papua New Guinea', } \\
\text { Paraguay, Peru, Philippines, Romania, Russian Federation, Rwanda, Samoa, 'São Tomé and Príncipe', } \\
\text { Senegal, Serbia, 'Sierra Leone', 'Solomon Islands', Somalia, 'South Africa', 'South Sudan', 'Sri Lanka', 'St. } \\
\text { Lucia', 'St. Vincent and The Grenadines', Sudan, Suriname, Swaziland, 'Syrian Arab Republic', Tajikistan, } \\
\text { Tanzania, Thailand, Timor-Leste, Togo, Tokelau, Tonga, Tunisia, Turkey, Turkmenistan, Tuvalu, Uganda, } \\
\text { Ukraine, Uzbekistan, Vanuatu, Venezuela, Vietnam, 'Wallis and Futuna', 'West Bank and Gaza', Yemen, } \\
\text { Zambia, Zimbabwe }\end{array}$ \\
\hline
\end{tabular}

developed with support from our institutional librarian. Through the entire process of its development, we used the McGowan et al's checklist to assess the adequacy of our electronic search strategy. ${ }^{29}$ Search results from the implementation of our search strategy as implemented in Scopus is presented as part of this protocol (see online supplementary material S2).

The websites of governments, non-government organisations, UN agencies and institutions that we know may have done costing of maternal health services from our experience will be searched to identify relevant grey literature. Specifically, we will search Google Scholar, websites of LMIC Ministries of Health, Population Council, Averting Maternal Death and Disability, Guttmacher Institute, FP2020, Maternal Health Task Force, United Nations Children's Fund, United Nations Fund for Population and WHO. In addition to the automated search, we will search for other relevant articles by reviewing the reference lists of retrieved articles. If a study is found in the grey literature, which was later published in the peer-reviewed literature, the peer-reviewed version will be selected for the review.

For both peer-reviewed and grey literature sources, the search will be focused on retrieving articles published from January 2000 to June 2019, as the costs data after this period are deemed to be more current and relevant for planning services in the SDG era. The search will be limited to studies published in English and French languages, which the authors understand. This search will be conducted independently by two authors (AB-T and I-OOA), with search results compared for completeness.

\section{Selection of studies}

Two co-authors (AB-T and I-OOA) will independently screen all retrieved records. Titles and abstracts will be screened for relevance and eligibility, based on the set inclusion/exclusion criteria (defined below). If titles or abstracts appear relevant, full-text will be subsequently reviewed to verify the relevance of the study for the review. Full-texts of retrieved articles will be stored in shared folders within an automated reference manager, Mendeley Desktop V.1.19.4 (Elsevier, Amsterdam, The Netherlands) for easy access for the review team. Any discrepancies regarding the relevance of studies for the review will be resolved through discussions with the senior coauthor (CAA). Reasons for decisions taken as regards inclusion or exclusion of studies will be documented. The inclusion/exclusion processes will also be reported as per the PRISMA reporting guidelines for systematic reviews. ${ }^{27}$

\section{Eligibility criteria}

Inclusion criteria

Full (cost minimisation, cost-effectiveness, cost-utility and cost-benefit analyses) and partial (cost analysis, cost-description studies and cost-outcome studies) economic evaluations of any or a combination of the maternal health services captured along the continuum of care as defined by Kerber et al. ${ }^{12}$ (ANC, skilled birth attendance, EmOC (including injectable antibiotics, injectable oxytocics, injectable anticonvulsants, manual removal of placenta, removal of retained products, assisted vaginal delivery, caesarean section and blood transfusion), PNC and family planning $)^{12}$ will be considered in this review. 
Full and partial economic evaluation studies have been selected as both typically report cost data, ${ }^{30}$ which is the focus of this review. Articles published in the peer-review or grey literature after year 2000 will be included if these present primary data on cost of any of the maternal health services provided to women regardless of the level of care (primary, secondary or tertiary levels) and collected from one or multiple LMIC(s), as defined by the World Bank. ${ }^{21}$ Studies published year 2000 onwards are deemed most relevant for the post-2015 era of the SDGs.

\section{Exclusion criteria}

Commentaries, editorials, letters that only broadly discuss the cost of providing maternal health services, as well as other reviews, will be excluded. In addition, articles will be excluded if the maternal health services are provided by individuals who are not legally allowed to provide such services in the country of study based on published national policy guidelines. In addition, health services that are part of the continuum of care but focused on newborn, children or adolescents ${ }^{12}$ will be excluded.

\section{Quality assessment of included studies}

The 24-item Consolidated Health Economic Evaluation Reporting Standards (CHEERS) checklist has typically been used for assessing the quality of reporting of the full economic evaluations. ${ }^{31}$ However, as many of the studies that will be included in this review may be purely cost analyses, which are a form of partial economic evaluations, ${ }^{30}$ an adapted quality assessment tool, which incorporates the relevant cost-focused criteria in the CHEERS checklist and those in the British Medical Journal Economic Evaluation Working Party, ${ }^{32}$ will be used. This choice is based on insight from quality assessments that have been conducted in similar systematic reviews that focused on cost of services. ${ }^{334}$

Specifically for this review, quality criteria to be used for assessment will include completeness of the title and abstract (or executive summary in the case of grey literature reports), clarity on the broad context for the study and study question, description of characteristics of the population, costs perspective used and time horizon, description of methods used to estimate costs, report on dates of the estimated costs and unit costs as well as methods used in converting costs into a common currency base and the exchange rate. In addition, the review will assess presentation of key study findings including a detailed breakdown of costs incurred, description of how conclusions were reached, discussion of study limitations and the generalisability of the findings and how the findings fit with current knowledge. ${ }^{31} 35$

For each item, a score of 1 will be awarded if the criterion is fully met, 0.5 , if partially met, 0 , if not met or if only minimal information was provided, and NA if not applicable. The total score achieved across all the criteria will be subsequently summed-up and converted to percentages. As has been done in other similar reviews, ${ }^{3336}$ studies with $75 \%$ or more criteria fully met will be classified as high quality, $50 \%-74 \%$ as average quality and $<50 \%$ as poor quality. Each included study will be assessed independently by two coauthors (AB-T and MA).

\section{Data extraction}

We will use two predeveloped Microsoft Excel forms focused on quality assessment and evidence synthesis to extract data for this review. For all included articles, we will collect data on the article description (authors, year of publication, article title, journal), study setting (country of study, country of organisation conducting study, characteristics of women receiving maternal health services who were surveyed or for whom costs data were collected, perspective of costing (health system, government or societal), study design (cost analysis, partial economic evaluation, full economic evaluation or nested in another study), costing of maternal health services (intervention(s) costed, costing method used (top-down or expenditure approach that involves breaking down total cost into component costs $\left(\mathrm{C}_{\text {Total }}=>\mathrm{C}_{1}+\mathrm{C}_{2}+\mathrm{C}_{3}\right)$ vs bottom-up or ingredient approach that involves building-up the component/ingredient cost to estimate the total cost $\left(\mathrm{C}_{1}\right.$ $\left.+\mathrm{C}_{2}+\mathrm{C}_{3}=>\mathrm{C}_{\text {Total }}\right)$ ), time frame, facility type (health centre, hospital), facility ownership (private, public or mission), number of facilities, component of cost included (eg, start-up cost, running cost, cost of labour, equipment, medicines, supplies and for those who do, opportunity $\operatorname{cost}^{30}$ and so on), year of costs data, currency and discount rate) as well as findings reported (including total service cost estimates per time period or where reported, cost per client visit). For articles which take a societal perspective and report the cost of utilising services, we will collect non-health facility-related costs such as transport. Guidance on costs data to be collected were sought from a previous review ${ }^{20}$ and an expert opinion. ${ }^{35}$

Data extraction will be conducted independently by two of the coauthors (AB-T and I-OOA) independently and then checked for accuracy by a third reviewer (OB-T). To minimise inconsistency between reviewers, we will conduct training and calibration exercises using the data extraction form prior to the commencement of the systematic review. In cases where data are missing from articles and not publicly available, we will make attempts to contact the study authors directly via the contact information provided in the study or report, or by using portals such as ResearchGate and LinkedIn.

\section{Data synthesis}

Characteristics of included studies will be summarised, and cost data provided by the authors will be collated within Microsoft Excel. Using a subgroup analysis, the different costs items associated with each service (medicines and supplies, equipment and labour costs) will be identified. Articles that include lump costs that cannot be disaggregated into service categories within the continuum of care pathway (ie, ANC, skilled birth attendance, EmOC, PNC and family planning $)^{12}$ will be analysed separately. Opportunity costs will be excluded 
before totalling the direct financial cost of each service, as not all costing studies typically include it. ${ }^{33}$ Comparison of service costs across countries will be performed. To allow ease of cost comparisons, purchasing power parity (PPP) conversion factors ${ }^{37}$ will be used to convert the local currency of the country in which the study was conducted to international dollar (I\$) equivalents for the reported year of cost data collection. ${ }^{38}$ Costs reported in US dollars using 'market exchange rates' will first be converted to local currency for the year the costing was done, using official OANDA Corporation exchange rates before being converted to I\$ using PPP factors. ${ }^{39} \mathrm{PPP}$, as opposed to market exchange rates, allows hypothetical estimation of the amount it would cost to purchase the same market basket of goods in various countries if their currencies were at par. $^{38}$ Based on these newly calculated I\$ equivalents, the unit cost per service will be calculated.

In addition, we will conduct a sensitivity analysis by repeating our analysis to include studies that only partly failed to meet our inclusion criteria. ${ }^{40}$ Risk of bias analysis will not be performed for this systematic review as it is focused only on costs and not effectiveness metrics, which would be required for such analysis. ${ }^{41}$

Finally, we will compare costs from each country and try to explain the reasons for any observed similarities and differences. Where we find that it will not be possible to pool some findings together due to methodological heterogeneity, we will conduct a narrative synthesis of the available information. In doing this, relevant country-specific issues related to delivering and utilising maternal health services in the individual study countries will be highlighted and used to explain findings. In addition, in line with global guidance for conducting economic evaluations, ${ }^{42}$ by implementing targeted searches of the literature (peer-reviewed and grey literature), we will attempt to identify the population contextual and intervention design characteristics that help support lower costs (cost savings) and improve the efficiency of maternal health service provision in LMICs. Furthermore, where possible, we will highlight the major cost drivers in providing maternal health services, as identified by the authors of the studies included in our review and/or based on our analysis which will show the largest component cost attributable to each service. These findings will constitute critical lessons that could be transferred from one LMIC to another.

\section{Ethics and dissemination}

No ethical approval will be required, as this review is based on already published data and does not involve interaction with human subjects. The plan for dissemination, however, is to publish the findings of the review in a peer-reviewed journal and present findings at highlevel international conferences that engage the most pertinent stakeholders. The proposed systematic review will provide a detailed summary of available evidence on costs maternal health services across the continuum of care and will complement evidence from modelled costing analysis conducted to estimate projected costs of achieving the SDG targets in LMICs. ${ }^{43}$

\section{DISCUSSION}

This protocol has been rigorously developed and designed specifically to assess the cost of maternal health services in LMICs. Given the limited recent evidence of cost associated with providing these critical services, findings from the review will be critical for researchers, policy-makers, government and non-governmental organisations for planning maternal and newborn health services in LMICs. If protocol modifications are required, the authors will include the detailed description of any changes along with a justification during the publication of the review.

Clearly, in the era of the SDGs in which the focus is to ensure that 'no one is left behind' including in terms of receiving critical healthcare, ${ }^{3}$ require up-to-date information on the costs associated with these services. This systematic review will be a one-stop shop for such data.

Contributors $A B-T$ conceived the review. AB-T, OB-T and CAA designed the review. I-OOA refined the review design. AB-T and AM were involved in the initial drafting of the manuscript. All authors were involved in subsequent draft manuscript reviews and updates and approved the final version of this protocol.

Funding The authors have not declared a specific grant for this research from any funding agency in the public, commercial or not-for-profit sectors.

Competing interests None declared.

Patient consent for publication Not required.

Provenance and peer review Not commissioned; externally peer reviewed.

Open access This is an open access article distributed in accordance with the Creative Commons Attribution Non Commercial (CC BY-NC 4.0) license, which permits others to distribute, remix, adapt, build upon this work non-commercially, and license their derivative works on different terms, provided the original work is properly cited, appropriate credit is given, any changes made indicated, and the use is non-commercial. See: http://creativecommons.org/licenses/by-nc/4.0/.

\section{REFERENCES}

1. Alkema L, Chou D, Hogan D, et al. Global, regional, and national levels and trends in maternal mortality between 1990 and 2015, with scenario-based projections to 2030: a systematic analysis by the un maternal mortality estimation Inter-Agency group. Lancet 2016;387:462-74.

2. Blencowe H, Cousens $\mathrm{S}$, Jassir FB, et al. National, regional, and worldwide estimates of stillbirth rates in 2015, with trends from 2000 : a systematic analysis. Lancet Glob Health 2016;4:e98-108.

3. United Nations. Sustainable development goals: 17 goals to transform our world. sustain. DEV. goals, 2016. Available: http:// www.un.org/sustainabledevelopment/sustainable-developmentgoals/ [Accessed 9 Aug 2019].

4. United Nations General Assembly. The road to dignity by 2030: ending poverty, transforming all lives and protecting the planet: synthesis report of the Secretary-General on the post-2015 sustainable development agenda. In: 69th session of the General Assembly. New York: United Nations, 2014.

5. Kuhnt J, Vollmer S. Antenatal care services and its implications for vital and health outcomes of children: evidence from 193 surveys in 69 low-income and middle-income countries. BMJ Open 2017;7:e017122.

6. Paxton A, Maine D, Freedman L, et al. The evidence for emergency obstetric care. International Journal of Gynecology \& Obstetrics 2005;88:181-93.

7. Singh A, Pallikadavath S, Ram F, et al. Do antenatal care interventions improve neonatal survival in India? Health Policy Plan 2014;29:842-8. 
8. Yakoob MY, Ali MA, Ali MU, et al. The effect of providing skilled birth attendance and emergency obstetric care in preventing stillbirths. BMC Public Health 2011;11(Suppl 3):S7.

9. Ni Bhuinneain GM, McCarthy FP. A systematic review of essential obstetric and newborn care capacity building in rural sub-Saharan Africa. BJOG: Int J Obstet Gy 2015;122:174-82.

10. Ahmed S, Li Q, Liu L, et al. Maternal deaths averted by contraceptive use: an analysis of 172 countries. Lancet 2012;380:111-25.

11. Kikuchi K, Ansah EK, Okawa S, et al. Effective linkages of continuum of care for improving neonatal, perinatal, and maternal mortality: a systematic review and meta-analysis. PLoS One 2015;10:e0139288.

12. Kerber KJ, de Graft-Johnson JE, Bhutta ZA, et al. Continuum of care for maternal, newborn, and child health: from slogan to service delivery. The Lancet 2007;370:1358-69.

13. Onarheim $\mathrm{KH}$, Iversen JH, Bloom DE. Economic Benefits of Investing in Women's Health: A Systematic Review. PLoS One 2016;11:e0150120.

14. Starbird E, Norton M, Marcus R. Investing in family planning: key to achieving the sustainable development goals. Glob Health Sci Pract 2016;4:191-210.

15. Adam T, Lim SS, Mehta S, et al. Cost effectiveness analysis of strategies for maternal and neonatal health in developing countries. BMJ 2005;331.

16. Johns B, Baltussen R, Hutubessy R. Programme costs in the economic evaluation of health interventions. Cost Effective Resour Alloc 2003;1.

17. Baltussen R, Adam T, et al, World Health Organization,. Making choices in health: who guide to cost-effectiveness analysis. Geneva: World Health Organization, 2003. http://www.who.int/choice/ publications/p_2003_generalised_cea.pdf

18. World Health Organization. Cost effectiveness and strategic planning (WHO-CHOICE). Health Econ. 2018;:Cost effectiveness and strategic planning (WHO-CHO. Available: http://www.who.int/choice/costeffectiveness/en/ [Accessed 9 Aug 2019].

19. Stenberg K, Lauer JA, Gkountouras G, et al. Econometric estimation of WHO-CHOICE country-specific costs for inpatient and outpatient health service delivery. Cost Eff Resour Alloc 2018;16.

20. Borghi J. What is the cost of maternal health care and how can it be financed? In: Brouwere D V, Van Lerberghe W, eds. Safe motherhood strategies: a review of the evidence. 2nd edn. Antwerp, Belgium: ITGPress, 2001: 243-92. http://hdl.handle.net/10390/2661

21. World Bank. World bank country and lending groups. data, 2018. Available: https://datahelpdesk.worldbank.org/knowledgebase/ articles/906519 [Accessed 9 Aug 2019].

22. Ebu NI, Gross J. Factors influencing access to antenatal services and delivery care in sub-Saharan Africa. Afr J Midwifery Womens Health 2015;9:58-65.

23. Banke-Thomas A, Madaj B, Kumar S, et al. Assessing valuefor-money in maternal and newborn health. BMJ Glob Health 2017;2:e000310.

24. Nixon J, Khan KS, Kleijnen J. Summarising economic evaluations in systematic reviews: a new approach. BMJ 2001;322:1596-8.

25. Carande-Kulis VG, Maciosek M V, Briss PA, et al. Methods for systematic reviews of economic evaluations for the guide to community preventive services. Am J Prev Med 2000;18:75-91.

26. Moher D, Shamseer L, Clarke M, et al. Preferred reporting items for systematic review and meta-analysis protocols (PRISMA-P) 2015 statement. Syst Rev 2015;4:1.
27. Moher D, Liberati A, Tetzlaff J, et al. Preferred reporting items for systematic reviews and meta-analyses: the PRISMA statement. PLoS Med 2009;6:e1000097.

28. Wilczynski NLet al. Optimal search strategies for detecting health services research studies in MEDLINE. Can Med Assoc $J$ 2004;171:1179-85.

29. McGowan J, Sampson M, Lefebvre C. An evidence based checklist for the peer review of electronic search strategies (press EBC). Evid Based Libr Inf Pract 2010;5:149.

30. Drummond MF, Sculpher MJ, Claxton K, et al. Methods for the economic evaluation of health care programmes. Oxford: Oxford University Press, 2015.

31. Husereau D, Drummond M, Petrou S, et al. Consolidated health economic evaluation reporting standards (cheers) statement. BJOG 2013;120:765-70.

32. Drummond MF, Jefferson TO. Guidelines for authors and peer reviewers of economic submissions to the BMJ. BMJ 1996;313:275-83.

33. Banke-Thomas A, Wilson-Jones M, Madaj B, et al. Economic evaluation of emergency obstetric care training: a systematic review. BMC Pregnancy Childbirth 2017:17:403.

34. Barber JA, Thompson SG. Analysis and interpretation of cost data in randomised controlled trials: review of published studies. BMJ 1998;317:1195-200.

35. Stone $G$, Hutchinson $A$, Corso $P$, et al. Understanding and Using the Economic Evidence. In: Zaza S, Briss PA, Harris KW, et al, eds. The guide to community preventive services. New York: Oxford University Press, 2005: 449-63.

36. Mangham-Jefferies L, Pitt C, Cousens S, et al. Cost-Effectiveness of strategies to improve the utilization and provision of maternal and newborn health care in low-income and lower-middleincome countries: a systematic review. BMC Pregnancy Childbirth 2014; $14: 243$.

37. United Nations. Purchasing power parities (ppp) conversion factor, local currency unit to international dollar. Ser. data, 2015.

38. Cheung Y-W. Purchasing Power Parity. In: Reinert KA, Rajan RS, Glass AJ, eds. The Princeton Encyclopedia of the World Economy. Princeton: Princeton University Press, 2009: 942-4.

39. OANDA Corporation. Currency converter. Curr. tools, 2018 Available: https://www.oanda.com/currency/converter/ [Accessed 9 Aug 2019].

40. Higgins JP, Green S, The Cochrane Collaboration. Cochrane handbook for systematic reviews of interventions. Version 5. London: The Cochrane Collaboration, 2011. http://handbook-5-1.cochrane. org/

41. Sterne JAC, Sutton AJ, loannidis JPA, et al. Recommendations for examining and interpreting funnel plot asymmetry in meta-analyses of randomised controlled trials. BMJ 2011;343:d4002.

42. Gomersall JS, Jadotte YT, Xue Y, et al. Conducting systematic reviews of economic evaluations. Int J Evid Based Healthc 2015;13:170-8.

43. Stenberg K, Hanssen O, Edejer TT-T, et al. Financing transformative health systems towards achievement of the health sustainable development goals: a model for projected resource needs in 67 low-income and middle-income countries. Lancet Glob Health 2017;5:e875-87. 